\title{
Reply to: COVID-19, children, and schools: overlooked and at risk
}

Dr Alasdair PS Munro ${ }^{1,2}$, Dr Asha C Bowen ${ }^{3,4}$, Dr Muge Cevik ${ }^{5,6}$

1. NIHR Southampton Clinical Research Facility and NIHR Southampton Biomedical Research Centre, University Hospital Southampton NHS Foundation Trust, Southampton, Hampshire, UK

2. Faculty of Medicine and Institute for Life Sciences, University of Southampton, Southampton, Hampshire, UK

3. Department of Infectious Diseases, Perth Children's Hospital, Nedlands, Western Australia, Australia

4. Wesfarmers Centre of Vaccines and Infectious Diseases, Telethon Kids Institute, Nedlands, Western Australia, Australia

5. National Health Service Lothian Infection Service, Regional Infectious Disease unit and Specialist Virology Laboratory, Royal Infirmary of Edinburgh, United Kingdom

6. Infection and Global Health Research, School of Medicine, University of St Andrews, Fife, United Kingdom

We read with interest the opinion piece from Dr Hyde regarding school opening during the COVID-19 pandemic [1]. We have closely followed the international literature about SARS-CoV-2 transmission dynamics in children (aged 0-18 years) [2,3]. Evidence informed discussion about school attendance in the context of COVID-19 is a high priority and this complex subject requires weighted expert review of the literature to accurately inform policy. As paediatricians and infectious diseases specialists, we wish to highlight important considerations when interpreting the available data.

Schools (closures as well as mitigation measures) have been a major part of the collective discourse in 2020, and to suggest they have been overlooked is erroneous. School closures were one of the earliest non-pharmaceutical interventions employed globally, leaving 1.6 billion children without the educational and social benefits they provide [4]. Interest is intense among scientific and mass media in any potentially relevant data with many studies ongoing.

Interpretation of these studies require understanding about differences between young children, adolescents and younger adults as the evidence now suggests significantly lower risk of infection, severe disease and transmission for those under 10 years. The evidence from multiple household contact tracing studies which are unaffected by school closures demonstrate significantly lower secondary attack rates in younger children than adults [5]. This information has important implications in informing transmission risk.

Prior experience from influenza pandemics left many with strong beliefs regarding children's role in propagating community transmission, although evidence to date does not bear this out for SARSCoV-2. Both direct and indirect evidence exist of a cursory role of younger children, which although contrary to influenza, was similar for SARS and MERS.

While this report highlights health risks to children, data so far suggests the opposite; England had 4 deaths in children $<15$ years of age [6], compared to around 20 deaths from seasonal influenza annually in the same age group. 
The discussion on schools is complex, demanding nuanced and balanced scientific and media coverage that considers not only epidemiological questions but also public health, educational, developmental, wellbeing and social equity concerns $[2,4]$. Any contribution must be weighed against the immense costs of long-term school closures, especially for younger children and the disadvantaged in the long term.

[1] Hyde Z. COVID-19, children, and schools: overlooked and at risk. Med J Aust n.d.

[2] Levinson M, Cevik M, Lipsitch M. Reopening Primary Schools during the Pandemic. N Engl J Med 2020. doi:10.1056/nejmms2024920.

[3] Faust SN, Munro APS. It's Time to Put Children and Young People First During the Global COVID-19 Pandemic 2020. doi:10.1001/jamapediatrics.2020.4582.

[4] UNESCO. Adverse consequences of school closures 2020. https://en.unesco.org/covid19/educationresponse/consequences (accessed August 16, 2020).

[5] Goldstein E, Lipsitch M, Cevik M. On the effect of age on the transmission of SARS-CoV-2 in households, schools and the community. J Infect Dis 2020. doi:10.1093/infdis/jiaa691.

[6] Ladhani SN, Amin-Chowdhury Z, Davies HG, Aiano F, Hayden I, Lacy J, et al. COVID-19 in children: analysis of the first pandemic peak in England. Arch Dis Child 2020;0:archdischild2020-320042. doi:10.1136/archdischild-2020-320042. 